for each SEM level. To evaluate statistical significance and marginal contribution of each SEM level for explaining IV uptake, the full model was fitted (all variables from all 5 SEM levels). To determine joint statistical significance of variables of each SEM level, the model without respective SEM level was compared with the full model using likelihood ratio test. Additionally, marginal contribution of each SEM level was measured by relative reduction in magnitude of pseudo $R$ square.

Results For both men and women, older age groups (85 or more vs 65-69 age group; $\mathrm{PR}=1.59$ for men and $\mathrm{PR}=1.56$ for women); having 3 or more chronic conditions ( $\mathrm{PR}=1.39$ for men and $\mathrm{PR}=1.35$ for women); number of GP and outpatient visits in the previous 4 weeks were associated to higher IV uptake. For men, only 2 out of the 5 SEM levels were associated to IV uptake (individual and organizational). For women 3 levels were relevant for this preventive measure (individual, organizational and community). Main marginal contribution for explaining the IV uptake, came from individual $(17.9 \%$ and $16.3 \%)$ and organizational $(30.7 \%$ and $22.7 \%)$ levels for both men and women respectively.

Conclusion Besides individual characteristics, like age and health status - known determinants of IV uptake - this study highlights the importance of access and use of health care services for adoption of IV preventive measure. Moreover, it evidences a sex differential behaviour that should be accounted in the definition of the IV campaign strategy.

\section{P36 A STRATEGY TO IDENTIFY YOUNG CHILDREN WITH DEVELOPMENTAL DISABILITIES VIA PRIMARY CARE RECORDS}

SC Masefield*, SL Prady, KE Pickett. Department of Health Sciences, University of York, York, UK

\subsection{6/jech-2019-SSMabstracts. 187}

Background Electronic health records use clinical codes to classify disease and conditions, not disability (how impairment affects human function). Codes for the degree of disability are not routinely recorded alongside the diagnosis, unless part of the diagnostic code e.g. profound learning disability. Existing strategies identify conditions associated with disability, prioritising either identifying every person with possible or highly probable disability to limit type I (false positive) or type II (false negative) misclassification error.

In high income countries, $1-4 \%$ of children have developmental disabilities. They can be diagnosed before the age of five but, in practice, developmental delay is often diagnosed and the disabling condition (e.g. autism spectrum disorders or cerebral palsy) diagnosed when the child is older. Diagnoses of both delay/generalised developmental disorders and a disabling condition diagnosis could indicate disability severity. Is a sensitive or specific strategy or a combination of both necessary to obtain a realistic estimate of developmental disability prevalence in preschool children?

This study aimed to develop and compare strategies to identify children with possible and probable developmental disabilities diagnosed before the age of five in primary care data.

Methods Two case ascertainment strategies were developed and the primary care records of children in the Born in
Bradford (BiB) cohort study (from birth to their fifth birthday) searched: 1) to identify children with conditions associated with substantial developmental disability (autism spectrum disorders, Down syndrome and cerebral palsy and moderate-profound learning disability); and 2) to identify children with indicators of developmental disability (developmental delay, generalised developmental disorders, mild and unknown severity learning disability).

Results The combined UK prevalence of the disabling conditions is 417 per 10,000 children below age 18 . The prevalence in the study sample $(n=9,727)$ was 85 per 10,000 $(n=47$ autism spectrum disorders, $n=24$ Down syndrome, $\mathrm{n}=12$ cerebral palsy). None had moderate-profound learning disability. Half also had disability indicators $(53 \%, n=44)$. The prevalence of disability indicators was 450 per 10,000 $(n=438)$. Of those with only indicators $(n=394), 75.9 \%$ had a single indicator. The most common indicators in both the condition and indicator groups were speech delay, developmental delay and developmental language delay.

Conclusion Using only disabling condition clinical codes for case ascertainment via primary care data is likely to greatly underestimate disability prevalence in children under the age of five. Where independent disability verification is not possible, the number of disability indicators may reflect disability severity.

\section{P37 HOW CAN THE RECENT STALLING OF LIFE EXPECTANCY} GAINS IN SCOTLAND BE BEST EXPLAINED?

${ }^{1} \mathrm{G}$ McCartney*${ }^{2}$ IJ Ramsay, ${ }^{1} \mathrm{~J}$ Minton, ${ }^{3} \mathrm{C}$ Fischbacher, ${ }^{1} \mathrm{~L}$ Fenton. ${ }^{1}$ Public Health Science, NHS Health Scotland, Glasgow, UK; ${ }^{2}$ National Records of Scotland, Edinburgh, UK; ${ }^{3}$ Public Health and Intelligence, NHS National Services Scotland, Edinburgh, UK

\subsection{6/jech-2019-SSMabstracts. 188}

Background Annual gains in life expectancy in Scotland have been slower in recent years than in the previous two decades for males and females. Similar slowdowns or even reversals have been observed in England, Wales, Northern Ireland and the USA. This contribution explores the contribution of specific causes of death to the changes in mortality by age and cause for two time periods: $2000-02$ to $2012-14$ and 201214 to $2015-17$.

Methods Life expectancy at birth was calculated from death and population counts available from National Records of Scotland (NRS), disaggregated by five year age categories and by ICD-10 underlying cause of death. Arriaga's method of life expectancy decomposition was applied to produce estimates of the contribution of different age groups and underlying causes of death to life expectancy at birth in each of the two periods.

Results Life expectancy trends deteriorated after 2012-14 and life expectancy subsequently fell. The worsening trend involved increased inequalities as it was more profound with increasing area deprivation. Almost all age groups saw a worsening of trends in the later time periods and this was also seen across almost all causes of death. In particular, the previously observed rapid improvements in circulatory causes which benefited those aged 55-84 years most, more than halved. There were also absolute increases in mortality rates for those aged 35-49 years due in large part to increases in drugrelated deaths; and amongst those aged $90+$ years due to increased mortality from dementia/Alzheimer's. 
Conclusion Although there has been substantial focus up to now on the role that increased mortality amongst the oldest groups has played in the recent life expectancy trends, more attention needs to be paid to the role that the slowdown in improvement for circulatory causes across a much wider age group has played, as well as the importance of rising drugrelated deaths in younger adults. Future research should seek to explain the changes in mortality trends for all age groups and causes of death and policymakers should act to protect the social security system and public services that are likely to be part of the causes of the recent trends.

\section{P38 RISK AND PROTECTIVE FACTORS FOR PSYCHOTIC EXPERIENCES IN ADOLESCENCE: A POPULATION-BASED STUDY}

${ }^{1} \mathrm{E}$ McMahon*, ${ }^{1,2} \mathrm{P}$ Corcoran, ${ }^{3} \mathrm{H}$ Keeley, ${ }^{4} \mathrm{M}$ Clarke, ${ }^{5} \mathrm{H}$ Coughlan, ${ }^{6} \mathrm{D}$ Wasserman, ${ }^{7,8} \mathrm{C}$ Hoven, ${ }^{6} \mathrm{~V}$ Carli, ${ }^{9,10} \mathrm{M}$ Sarchiapone, ${ }^{5} \mathrm{M}$ Cannon. ${ }^{1}$ National Suicide Research Foundation, University College Cork, Cork, Ireland; ${ }^{2}$ School of Public Health, University College Cork, Cork, Ireland; ${ }^{3}$ Child and Adolescent Mental Health Services, Health Service Executive, Cork, Ireland; ${ }^{4}$ Depts of Psychology and Psychiatry, Royal College of Surgeons in Ireland, Dublin, UK; ${ }^{5}$ Dept of Psychiatry, Royal College of Surgeons in Ireland, Dublin, UK; ${ }^{6}$ National Centre for Suicide Research and Prevention of Mental III-Health (N, Karolinska Insitute, Stockholm, Sweden; ${ }^{7}$ Child and Adolescent Psychiatry, Columbia University, New York, USA; ${ }^{8}$ Child and Adolescent Psychiatry, New York State Psychiatric Institute, New York, USA; ${ }^{9}$ Dept of Medicine and Health Science, University of Molise, Campobasso, Italy; ${ }^{10}$ National Institute for Health, Migration and Poverty, Rome, Italy

\subsection{6/jech-2019-SSMabstracts. 189}

Background Psychotic experiences (PEs) are reported by a significant minority of adolescents and are associated with the development of schizophrenia and other psychiatric disorders in adulthood. Few modifiable protective factors have been identified to date. The aims of this study were to examine associations between a range of psychopathological, adverse life event, coping and social support factors and PEs in a general population sample of Irish adolescents.

Methods Cross-sectional data were drawn from the Irish centre of the Saving and Empowering Young Lives in Europe (SEYLE) study (German Clinical Trials Registry, DRKS00000214). Students were administered a classroombased self-report questionnaire and 973 adolescents, of whom 522 (53.6\%) were boys, participated. Psychotic experiences were assessed using the 7-item Adolescent Psychotic Symptom Screener.

Results Of the total sample, $81(8.7 \%)$ of the sample were found to be at risk of PEs. A wide range of factors from adverse life event, lifestyle and mental health domains had crude associations with PEs, while parental support was associated with lower prevalence of PEs. In multivariate analysis, independent associations were found between PEs and the number of adverse life events experienced (OR: 1.61; CI: 1.29-2.02; $\mathrm{p}<0.0005)$ as well as maladaptive/pathological internet use (OR: 2.60; CI: 1.15-5.89; $\mathrm{p}=0.02$ ). Positive parental support was associated with reduced risk of PEs after adjustment for established risk factors (OR: 0.40; CI: $0.18-$ $0.90 ; p=0.03)$ and so offers a potential protective role.

Conclusion These findings can inform the development of optimal interventions for adolescents at risk of psychopathology and their families.

\section{P39 MEAT, FRUIT AND VEGETABLE CONSUMPTION IN SUB-SAHARAN AFRICA: A SYSTEMATIC REVIEW AND META-REGRESSION}

${ }^{1}$ DO Mensah*, ${ }^{1} 0$ Oyebode, ${ }^{1}$ RA Nunes, ${ }^{2}$ R Lillywhyte. ${ }^{1}$ Health Sciences Division, Warwick Medical School, University of Warwick, Coventry, UK; ${ }^{2}$ School of Life Sciences, University of Warwick, Coventry, UK

\subsection{6/jech-2019-SSMabstracts. 190}

Background The dietary choices we make affect our personal health and have consequences for the environment, both of which have serious implications for the 2030 Sustainable Development Agenda. There is a strong consensus that cutting on meat and dairy products in favour of fruit and vegetables and other plant-based diets would offer dual health and environmental benefits. In global reviews, the literature on meat, fruit, and vegetable consumption in sub-Saharan Africa (SSA) is limited. It is therefore essential to quantify meat, fruit, and vegetable consumption in sub-Saharan African populations.

Methods We systematically searched six databases to identify studies reporting meat, fruit and/or vegetable consumption in sub-Saharan African populations. Using STATA SE version 15, random effects meta-regression analyses were used to test the effect of year of data collection and method of data collection on population meat, fruit, and vegetable consumption. We also tested any association between age, sex, urban/rural residence or a country's economic development, and population intake of meat, fruits and/or vegetables.

Results Richer SSA countries were likely to consume more meat $(B=36.76, p=0.04)$ and vegetables $(B=43.49, p=0.00)$ than poorer countries. Vegetable intake has increased dramatically over the last three decades from $\approx 10 \mathrm{~g}$ to $\approx 110 \mathrm{~g}$ $(\beta=4.43, p=0.00)$. Vegetable $(\beta=-25.48, p=0.00)$ consumption was higher in rural than urban residents. Although the trend of meat consumption has gone up $(\approx 25 \mathrm{~g}$ to $\approx 75 \mathrm{~g})$, the trend is non-significant $(\beta=0.63$, N.S.). Daily average per capita meat consumption was however above recommended $70 \mathrm{~g}$, while fruit and vegetable consumption remain below WHO's recommendation, though consumption of both fruit and vegetable has increased over the last three decades. No clear differences in consumption were noticed between sexes.

Conclusion While dietary changes in SSA may offer the large absolute benefits, consideration of the magnitude of dietary change, particularly increasing meat consumption, will need to occur to ensure policy and interventions support the reduction of under-nutrition and micronutrient deficiencies without worsening NCD prevalence and environmental impacts.

\section{P40 PREDICTING TYPE 2 DIABETES DEVELOPMENT AMONG PATIENTS IN GENERAL PRACTICE - A PROSPECTIVE ANALYSIS COMPARING METABOLIC SYNDROME DEFINITIONS AND COMPONENTS}

SR Millar*, CM Phillips, JM Harrington, IJ Perry. School of Public Health, University College Cork, Cork, Ireland

\subsection{6/jech-2019-SSMabstracts.191}

Background A definition of metabolic syndrome (MetS) has been recommended as a tool to help identify individuals at risk of developing type 2 diabetes. However, an agreed 